\title{
Theoretical Analysis of Thermodynamic Effect of Cavitation in Cryogenic Inducer Using Singularity Method
}

\author{
S. Watanabe, ${ }^{1}$ A. Furukawa, ${ }^{1}$ and Y. Yoshida ${ }^{2}$ \\ ${ }^{1}$ Department of Mechanical Engineering, Kyushu University, 744 Motooka, Nishi-ku, Fukuoka 819-0395, Japan \\ ${ }^{2}$ Space Transportation Propulsion Research and Development Center, Space Transportation Mission Directorate, \\ Japan Aerospace Exploration Agency, 1 Koganezawa, Kimigaya, Kakuda 981-1525, Japan
}

Correspondence should be addressed to S. Watanabe, fmnabe@mech.kyushu-u.ac.jp

Received 1 April 2008; Accepted 11 June 2008

Recommended by Sung Ro

\begin{abstract}
Vapor production in cavitation extracts the latent heat of evaporation from the surrounding liquid, which decreases the local temperature, and hence the local vapor pressure in the vicinity of cavity. This is called thermodynamic/thermal effect of cavitation and leads to the good suction performance of cryogenic turbopumps. We have already established the simple analysis of partially cavitating flow with the thermodynamic effect, where the latent heat extraction and the heat transfer between the cavity and the ambient fluid are taken into account. In the present study, we carry out the analysis for cavitating inducer and compare it with the experimental data available from literatures using Freon R-114 and liquid nitrogen. It is found that the present analysis can simulate fairly well the thermodynamic effect of cavitation and some modification of the analysis considering the real fluid properties, that is, saturation characteristic, is favorable for more qualitative agreement.
\end{abstract}

Copyright (C) 2008 S. Watanabe et al. This is an open access article distributed under the Creative Commons Attribution License, which permits unrestricted use, distribution, and reproduction in any medium, provided the original work is properly cited.

\section{INTRODUCTION}

Vapor production in cavitation extracts the latent heat of evaporation from the surrounding liquid, which decreases the local temperature, and hence the local vapor pressure in the vicinity of cavity. This is called a thermodynamic/thermal effect of cavitation. The thermodynamic effect of cavitation could be ignored for usual applications especially with water at ambient temperature, but is much more important for cryogenic fluids such as liquid oxygen and liquid hydrogen. For example, the suction performance of turbopump inducer for liquid propellant rocket engine is much better if operated with cryogenic fluids than cold water (Yoshida et al. [1]); the development of cavity is suppressed because of local vapor pressure depression due to the thermodynamic effect of cavitation. Recently, Franc et al. [2] have visually observed the thermodynamic effect in a turbopump inducer by using Freon R-114 as a working fluid, and succeeded in evaluating the effective temperature depression and Stepanoff's B-factor (Stepanoff [3]) through the comparison of cold water and R-114. Yoshida et al. [1] have measured the casing wall pressure distribution in an inducer with liquid nitrogen, and estimated the cavity volume from the low pressure region in the casing wall pressure distribution. Then, they have evaluated the effective temperature depression and B-factor by comparing the cavitation number which gives the same cavity length between cold water and liquid nitrogen.

Many theoretical/numerical studies have been done to clarify the thermodynamic effect of cavitation. Focusing on the recent studies, Kato [4] proposed a simple model expressing the heat flow around the sheet cavity by one-dimensional partial differential equation of unsteady heat conduction, and Tokumasu et al. [5] investigated the thermodynamic effect on closed sheet cavities by combining RANS simulation with Kato's model. Tani and Nagashima [6] simulated the cavitating flow around a hydrofoil with cryogenic fluids by the bubbly flow model based on the Rayleigh Plesset equation. Hosangadi et al. [7] have developed the compressible two-phase flow analysis considering the evaporation and the condensation processes, and compared their results with experiments using various test models and several cryogens (Hord [8]).

We have developed a simple analysis of unsteady cavitating flow combining a free streamline theory and a singularity method, and succeeded in simulating the cavitation instabilities of hydrofoil (Watanabe et al. [9]) as well as those 


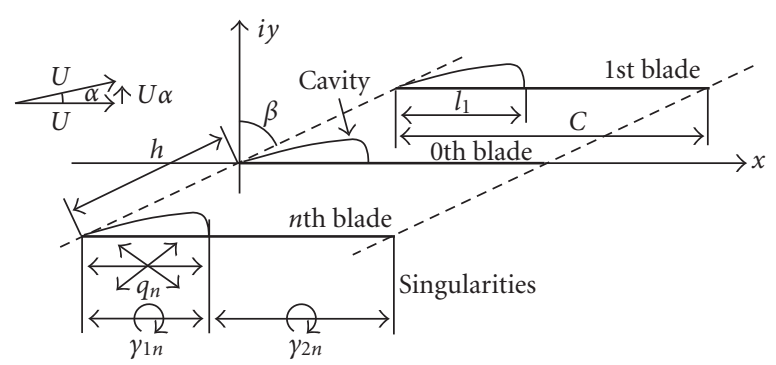

FIGURE 1: Model for present analysis.

of cascade such as rotating cavitation and cavitation surge (Watanabe et al. [10]). More recently, we have constructed an analytical method of the thermodynamic effect on steady, partially cavitating flow (Watanabe et al. [11]). In this work, a singularity analysis is combined with a heat transfer model proposed by Kato [4] and described above.

In the present study, we apply our analysis for a cavitating flow of two different working fluids, Freon R-114 and liquid nitrogen, in a cascade and compare the results with the experimental ones in a turbopump inducer reported by Franc et al. [2] and Yoshida et al. [1]. Some modification of the analysis is described, which seems to be necessary to obtain more qualitative agreement with the experiments using cryogenic fluids.

\section{FUNDAMENTAL FLOW FIELD}

We consider a flat plate cascade with the chordlength $C$, spacing $h$, the stagger angle $\beta$, and the number of blade $N$ as shown in Figure 1. We assume that the flow far upstream is uniform with the magnitude $U$ and the angle of attack $\alpha$. Each blade is represented by bound vortex distributions $\gamma_{n}(\xi)[n=0,1, \ldots, N-1]$, and the cavity with length $l_{n}$ which develops on the suction surface of each blade is represented by source distributions $q_{n}(\xi)[n=0,1, \ldots, N-1]$. Then the complex conjugate velocity is expressed by the following equation:

$$
\begin{aligned}
w(z)=u-i v & \\
=U e^{-i \alpha}+\frac{1}{2 \pi} \sum_{n=0}^{N-1} & {\left[\int_{0}^{l_{n}} q_{n}(\xi) \cdot\left\{f_{n}(z, \xi)+\frac{\pi}{N h} e^{i \beta}\right\} d \xi\right.} \\
& +i \int_{0}^{l_{n}} \gamma_{1 n}(\xi) \cdot\left\{f_{n}(z, \xi)+\frac{\pi}{N h} e^{i \beta}\right\} d \xi \\
& \left.+i \int_{l_{n}}^{C} \gamma_{2 n}(\xi) \cdot\left\{f_{n}(z, \xi)+\frac{\pi}{N h} e^{i \beta}\right\} d \xi\right],
\end{aligned}
$$

$f_{n}(z, \xi)=\frac{\pi}{N h} e^{-i(\pi / 2-\beta)} \cdot \cot \left[\frac{\pi}{N h}(z-\xi) e^{-i(\pi / 2-\beta)}-\frac{n \pi}{N}\right]$.

We divide the velocity components into the uniform velocity $U$ and the deviation $\left(u_{s}, v_{s}\right)$, that is,

$$
u=U+u_{s}, \quad v=U \alpha+v_{s}
$$

In the present study, we linearize the equations under the assumptions of the small angle of attack $\alpha \ll 1$ and the small velocity deviations $\left|u_{s}\right|,\left|v_{s}\right| \ll U$.

\section{BOUNDARY AND COMPLEMENTARY CONDITIONS}

We assume that the cavity is sufficiently thin so that all boundary conditions are applied on the blade suction surface. In the following sections, boundary and complementary conditions applied are described for $n$th blade.

\subsection{Boundary condition on cavity surface}

We assume that the pressure on the cavity surface is equal to the vapor pressure, which is locally different due to the temperature depression around the cavity surface under the presence of the thermodynamic effect of cavitation. Integrating the linearized momentum equation in the $x$ direction, we obtain the following equation:

$$
\frac{u_{c s n}(x)}{U \alpha}=\frac{\sigma_{n}(x)}{2 \alpha},
$$

where $u_{c s n}(x)$ denotes the velocity on the cavity surface at $x$. The local cavitation number $\sigma_{n}(x)$ has been defined using the local vapor pressure $p_{v n}(x)$,

$$
\sigma_{n}(x)=\frac{p_{\infty}-p_{v n}(x)}{\rho_{L} U^{2} / 2},
$$

where $p_{\infty}$ denotes the pressure far upstream and $\rho_{L}$ denotes the density of liquid phase.

\subsection{Boundary condition on wetted surface}

We employ the following flow tangency condition on the wetted blade surfaces:

$$
\begin{aligned}
& \operatorname{Imag}\left[w\left\{n h e^{-i(\pi / 2-\beta)}+x-0 i\right\}\right]=0 \quad\left(0<x<l_{n}\right), \\
& \operatorname{Imag}\left[w\left\{n h e^{-i(\pi / 2-\beta)}+x \pm 0 i\right\}\right]=0 \quad\left(l_{n}<x<C\right) .
\end{aligned}
$$

\subsection{Kutta's condition}

We assume that the pressure difference across the blade vanishes at the trailing edge. This condition is simply expressed as follows:

$$
\gamma_{2 n}\left(l_{n}\right)=0
$$

\subsection{Cavity closure condition}

We employ the closed cavity model for its simplicity. The cavity thickness $\eta_{n}$ can be obtained by integrating the following kinematic boundary condition on the cavity surface:

$$
U \frac{d \eta_{n}}{d x}=q_{n}(x) .
$$

Imposing the zero cavity thickness at the trailing edge of cavity, we obtain the following cavity closure condition:

$$
\eta_{n}\left(l_{n}\right)=\frac{1}{U} \int_{0}^{l_{n}} q_{n}(\xi) d \xi=0 .
$$




\section{MODELING OF THERMODYNAMIC EFFECT}

In the previous section, we have described about the kinematic and dynamic boundary conditions, assuming that the local vapor pressure distribution along the cavity surface is known. To close the problem, we have to obtain the local vapor pressure distribution. Here, we model the thermodynamic effect of cavitation, using the following heat conduction model for the liquid flow around the cavity and the evaporation model expressing the heat flux across the cavity surface due to evaporation.

\subsection{Heat conduction model}

We assume that the heat conduction in the main flow direction $(x)$ is negligibly small, compared to that in the direction $(y)$ normal to the cavity surface. The temperature increase (decrease) due to the formation of thin cavity $\Delta T_{n}$ should satisfy the following linearized energy equation for inviscid flow:

$$
\frac{\partial \Delta T_{n}}{\partial t}+U \frac{\partial \Delta T_{n}}{\partial x}=\varepsilon a \frac{\partial^{2} \Delta T_{n}}{\partial y^{2}}
$$

where a denotes a thermal diffusivity. Kato's turbulent diffusivity factor $\varepsilon$ (Kato [4]) has been employed to take account of the enhancement of thermal diffusion due to the turbulent flow around the cavity.

Temperature difference $\Delta T_{n}$ between far upstream and the cavity surface is expressed as follows:

$$
\Delta T_{n}=T_{n}(x, 0)-T_{\infty}=\alpha T_{\infty} C_{T n}(x, 0),
$$

where we have assumed that the temperature difference $\Delta T_{n}$ is sufficiently small and changes linearly with the change of the incidence $\alpha$. Substituting the above equation into (10), we obtain the following equation:

$$
U \frac{\partial C_{T n}}{\partial x}=\varepsilon a \frac{\partial^{2} C_{T n}}{\partial y^{2}}
$$

We can find that this equation is equivalent to onedimensional partial differential equation of unsteady heat conduction. Assuming no temperature depression at the leading edge of cavity $(x=0)$, we can analytically integrate the above equation using the normalized temperature increase along the cavity surface $C_{\theta n}(x)$ :

$$
C_{T n}(x, y)=\frac{y}{2 \sqrt{\pi K_{P}}} \int_{0}^{x} C_{\theta n}(\xi) \frac{\exp \left(-y^{2} / 4 K_{P}(x-\xi)\right)}{(x-\xi)^{3 / 2}} d \xi,
$$

where $K_{P}$ is defined here as $K_{P}=\varepsilon a / U$. Then, the temperature gradient normal to the cavity surface is derived as

$$
\lim _{y \rightarrow 0} \frac{\partial C_{T n}(x, y)}{\partial y}=-\frac{1}{\sqrt{\pi K_{P}}} \int_{0}^{x} \frac{d C_{\theta n}(\xi)}{d \xi} \frac{1}{(x-\xi)^{1 / 2}} d \xi .
$$

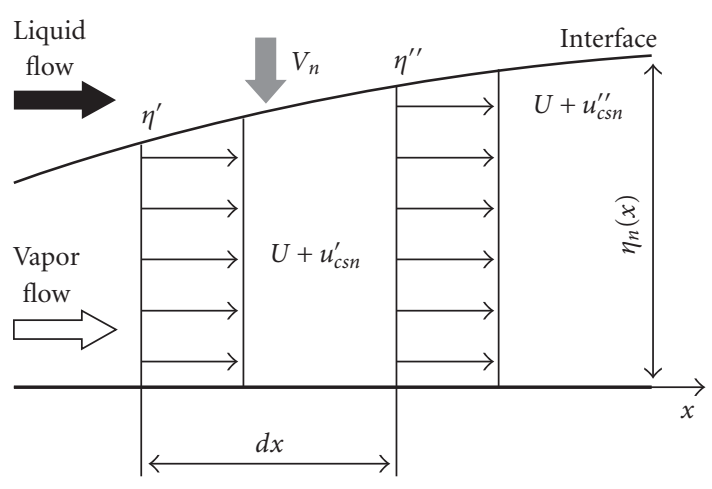

FIGURE 2: Control volume for continuity equation.

\subsection{Evaporation model}

We assume that the velocity inside the cavity is uniform in the $y$-direction which equals to the liquid velocity $U+u_{c s n}$ on the cavity surface as shown in Figure 2. Considering the continuity relation in the small control volume inside the cavity, we obtain the following equation using the local evaporation velocity $V_{n}$ of vapor phase:

$$
V_{n} d x=\left(U+u_{c s n}^{\prime \prime}\right) \eta_{n}^{\prime \prime}-\left(U+u_{c s n}^{\prime}\right) \eta_{n}^{\prime}=U d \eta_{n}
$$

From this equation, we can calculate the local evaporation velocity $V_{n}$. However, it is experimentally observed that, in general cases, the cavitation bubbles do not collapse completely at the trailing edge and some part of the cavity is flown away as a form of the cloud cavity shedding. The magnitude of the cloud cavity shedding becomes larger as the cavity becomes longer. Then, in order to take account of the effect of the cloud cavity shedding, we modify the evaporation velocity by multiplying the cavity length $\left(l_{n} / C\right)$, which has been known to give a good approximation in our previous study (Watanabe et al. [11]). Then, the heat flux due to the local latent heat of evaporation on the cavity surface can be expressed as follows:

$$
q_{T n}=-\rho_{V} L \frac{l_{n}}{C} V_{n}=-\rho_{V} L \frac{l_{n}}{C} U \frac{d \eta_{n}}{d x}=-\rho_{V} L \frac{l_{n}}{C} q_{n},
$$

where $L$ denotes the latent heat of evaporation and $\rho_{V}$ is the density of vapor phase. By using the expression $C_{q_{n}}=q_{n} / U \alpha$, which will be introduced in the next section, we obtain

$$
q_{T n}=-L \rho_{V} \frac{l_{n}}{C} U \alpha C_{q n}
$$

We can also calculate the local heat flux from the temperature gradient (14) as

$$
q_{T n}=-\left.\varepsilon \lambda \frac{\partial T_{n}}{\partial y}\right|_{y=0}=\frac{\varepsilon \lambda \alpha T_{\infty}}{\sqrt{\pi K_{P}}} \int_{0}^{x} \frac{d C_{\theta n}(\xi)}{d \xi} \frac{1}{(x-\xi)^{1 / 2}} d \xi,
$$


where $\lambda$ denotes the thermal conductivity for the liquid phase. By equating this equation with (17), we obtain the following one:

$$
\int_{0}^{x} \frac{d C_{\theta n}(\xi) / d \xi}{(x-\xi)^{1 / 2}} d \xi=-\left(\frac{l_{n}}{C}\right) \frac{L}{C_{P} T_{\infty}}\left(\frac{\rho_{V}}{\rho_{L}}\right) \sqrt{\frac{C U}{a}} \sqrt{\frac{\pi}{C \varepsilon}} C_{q n}
$$

where $C_{P}$ denotes the specific heat of liquid phase. As we can see from (17), the heat flux across the cavity surface is directly related to the source term $C_{q n}=q_{n} / U \alpha ; C_{q n}>0$ corresponds to the evaporation with the heat absorption, and $C_{q n}<0$ corresponds to the condensation with the heat generation. Then, we have heat absorption from the surrounding liquid near the leading edge and heat generation near the trailing edge of the cavity. In the present closed cavity model, the cavity terminates with a rapid condensation near the trailing edge, but it is again reasonable to imagine that some portion of vapor flows away forming a cloud cavitation and condensates far downstream of the cascade. Then, as we have done in our previous study (Watanabe et al. [11]), we hereafter take account only of the heat flux due to evaporation and neglect that due to condensation near the trailing edge of cavity.

\subsection{Vapor pressure}

We have assumed that the pressure on the cavity surface is equal to the vapor pressure, which is locally different due to the temperature depression around the cavity surface under the presence of the thermodynamic effect of cavitation. In order to relate the vapor pressure $P_{V n}(x)$ with the temperature $T_{n}(x)$, we apply the Clapeyron-Clausius equation as follows:

$$
\begin{aligned}
P_{V n}(x)-P_{V \infty} & =\frac{d P_{V}}{d T}\left(T_{n}(x, 0)-T_{\infty}\right) \\
& =\frac{L}{T_{\infty}} \frac{\rho_{V} \rho_{L}}{\rho_{L}-\rho_{V}}\left(T_{n}(x, 0)-T_{\infty}\right) \\
& =\rho_{V} L \alpha C_{T n}(x, 0),
\end{aligned}
$$

where we have assumed that $\rho_{V} \ll \rho_{L}$. Then the cavitation number based on the local vapor pressure $\sigma_{n}(x)$ defined in (5) can be expressed as follows:

$$
\frac{\sigma_{n}(x)}{2 \alpha}=\frac{\sigma}{2 \alpha}-\left(\frac{\rho_{V}}{\rho_{L}}\right) \frac{L}{U^{2}} C_{T n}(x, 0),
$$

where $\sigma=2\left(P_{\infty}-P_{v \infty}\right) / \rho_{L} U^{2}$ is a usual cavitation number based on the far upstream values.

\section{ANALYTICAL METHOD}

Discretization of singularities distributed along the blades and cavities are made in the same manner as Horiguchi et al. [12], where nodes are distributed more densely near the leading and trailing edges of the blades and cavities. The temperatures along the cavity surface are evaluated at the midpoints between each node as well as at the leading edge.
The control points, where boundary conditions are applied, are also placed at the midpoints between each node.

We define the normalized strength of singularities as follows:

$$
\begin{aligned}
C_{q n}(\xi) & =\frac{q_{n}(\xi)}{U \alpha}, \\
C_{\gamma 1 n}(\xi) & =\frac{\gamma_{1 n}(\xi)}{U \alpha}, \\
C_{\gamma 2 n}(\xi) & =\frac{\gamma_{2 n}(\xi)}{U \alpha} .
\end{aligned}
$$

Discretizing the boundary and complementary conditions (4)-(9), (19), (21), we obtain the following set of linear equations:

$$
\begin{aligned}
& {\left[A\left(l_{n}\right)\right]\{Q\}=\{B\}} \\
& \{Q\}= \\
& \quad\left\{C_{q n}(\xi), \ldots, C_{\gamma 1 n}(\xi), \ldots, C_{\gamma 2 n}(\xi), \ldots, C_{\theta n}(\xi), \ldots, \frac{\sigma}{2 \alpha}\right\}^{T},
\end{aligned}
$$

where $A\left(l_{n}\right)$ and $B$ are a coefficient matrix and a constant vector, respectively. From this equation, we can obtain the cavity length $l_{n}$ as well as the other unknowns for the given value of $\sigma / 2 \alpha$.

\section{RESULTS AND DISCUSSIONS}

The present analysis treats the cavity on each blade individually, so that it can be applied to the analysis of cavitating flow with different cavity shapes for each blade such as alternate blade cavitation, which is known to occur for inducers with even blade count (Horiguchi et al. [12]). However, in the present paper, we concentrate on the cavitating flow with identical cavities for each blade.

\subsection{For Freon $\mathbf{R}-114$}

Figure 3 shows Franc's experimental results by using Freon R-114 and cold water as working fluids (Franc et al. [2]). As we can see from the plot of the cavity length against cavitation number (parameter) in Figure 3(a), the development of the cavity is suppressed for R114 with the higher temperature compared with those for cold water and R114 with the lower temperature due to the thermodynamic effect of cavitation. Franc et al. have estimated the effective temperature depression due to the thermodynamic effect of cavitation by using the following equation:

$$
\frac{1}{2} \rho_{L} U^{2}\left(\sigma_{c}-\sigma\right)=\frac{d P}{d T} \Delta T
$$

where $\sigma_{c}$ denotes the cavitation number without the thermodynamic effect. In cold water, the thermodynamic effect can usually be negligible, then the cavitation number in cold water is herein used for $\sigma_{c}$. Under the assumption that the flow around the cavity for the given cavity length is similar even in the different working fluids, we can estimate 


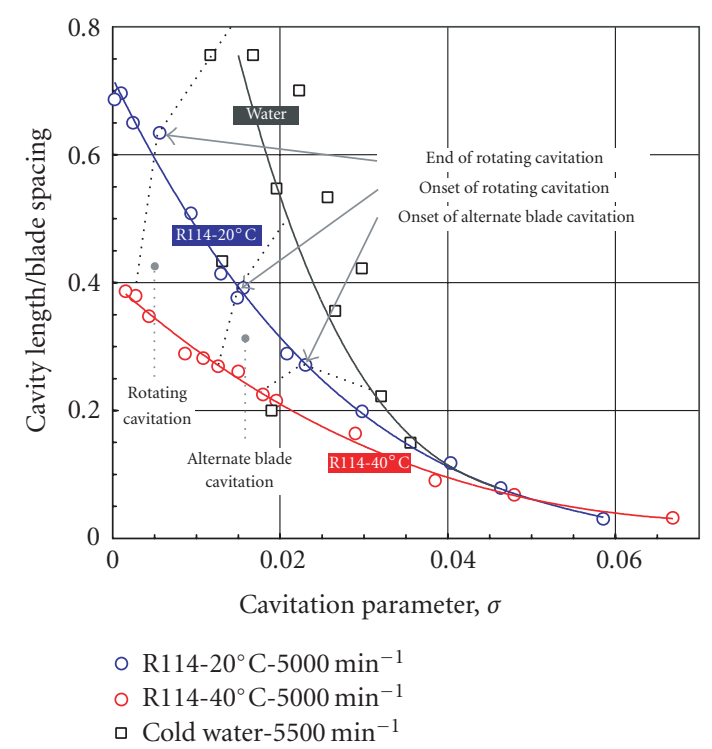

(a) Cavity length

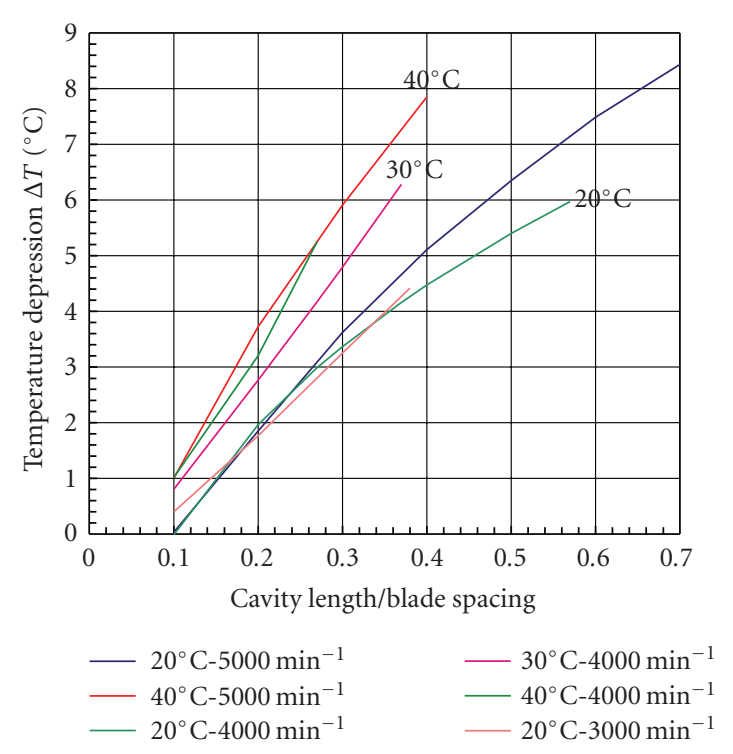

(b) Temperature depression

FIGURE 3: Experimental results in the case of R-114 and cold water (Franc et al. [2]).

the effective temperature depression via (24) by comparing the cavitation number with the same cavity length between cold water and the other fluid. We can see from Figure 3(b) that the temperature depression is more significant in the higher temperature cases with the higher rotational speed. The temperature depression becomes larger as the cavity becomes longer, which is simply because the amount of the evaporation is larger for longer cavitation.

Figure 4 shows the present analytical result for the R114 and cold water cases. The analysis has been done for the cascade with the stagger angle of $78.8^{\circ}$, the solidity of 2.0, and the dimensional chord length is $0.203 \mathrm{~mm}$ as a numerical configuration. The incidence angle and the main

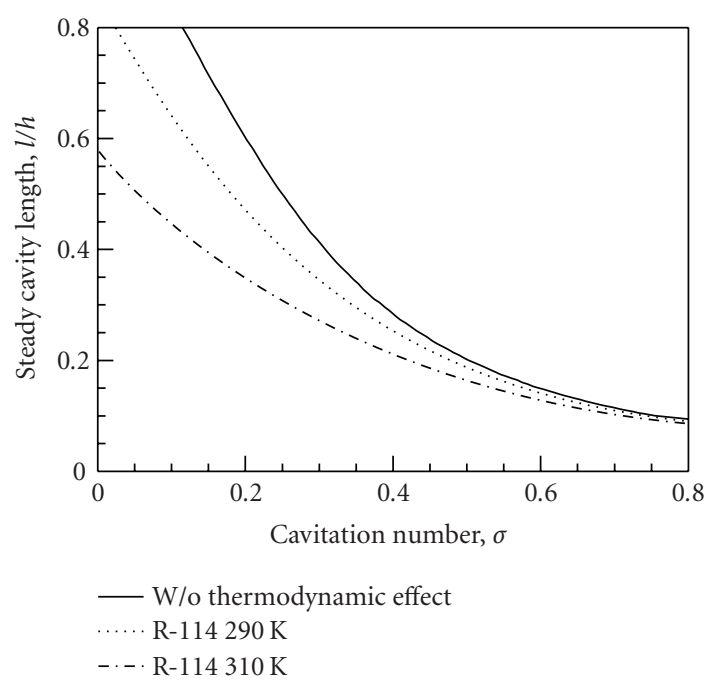

(a) Cavity length

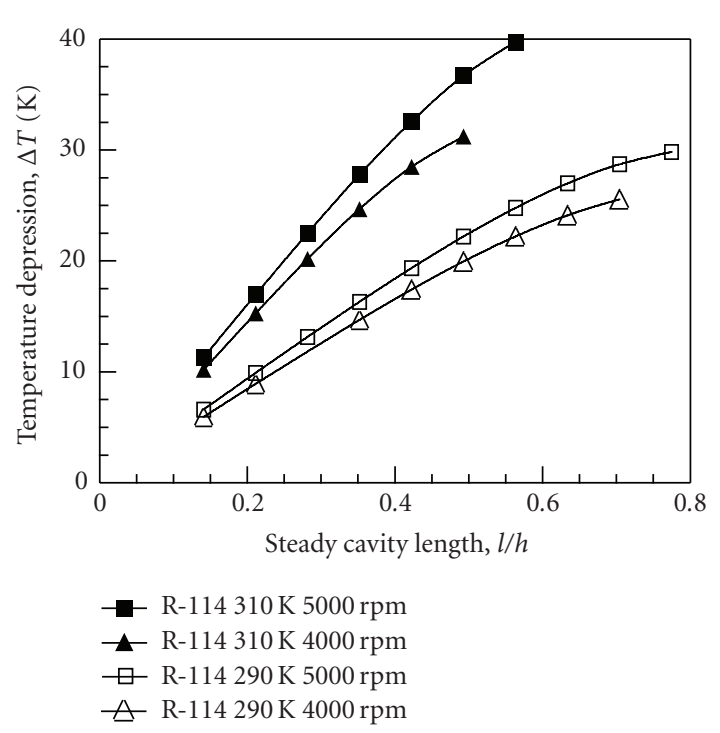

(b) Temperature depression

FIgURE 4: Numerical results in the case of R-114 and cold water.

flow velocity are chosen as $\alpha=4.32^{\circ}$ and $U=34.0 \mathrm{~m} / \mathrm{s}$ with the rotational speed of $5000 \mathrm{~min}^{-1}$. The turbulence diffusion factor $\varepsilon$ is unknown but is set to be 1000 , which gives comparable figures with the experimental ones. We can see from the comparisons between Figures 3 and 4 that the present analysis can simulate the phenomena fairly well; the thermodynamic effect is more apparent with the larger temperature depression for the higher temperature cases.

\subsection{For liquid nitrogen}

Figure 5 shows Yoshida's experimental results by using liquid nitrogen (LN2) with $80 \mathrm{~K}$ and cold water as a working fluid (Yoshida et al. [1]). In their experiments, they succeeded in measuring the cavity length indirectly from the pressure measurement, which is plotted in Figure 5(a). They also 


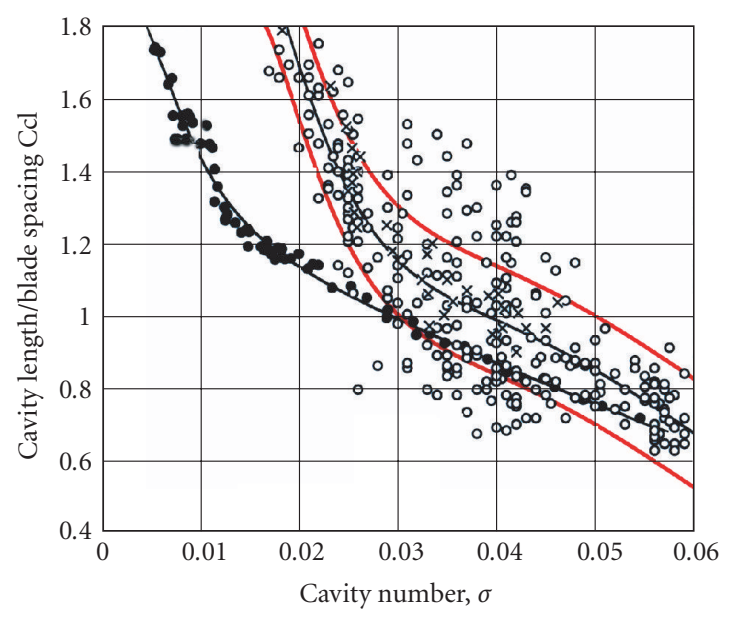

- Nitrogen $(80 \mathrm{~K})$

- Water $(296 \mathrm{~K})$ by visualization

$\times \quad$ Water $(296 \mathrm{~K})$ by pressure sensor

- Fit curve \pm standard deviation

(a) Cavity length

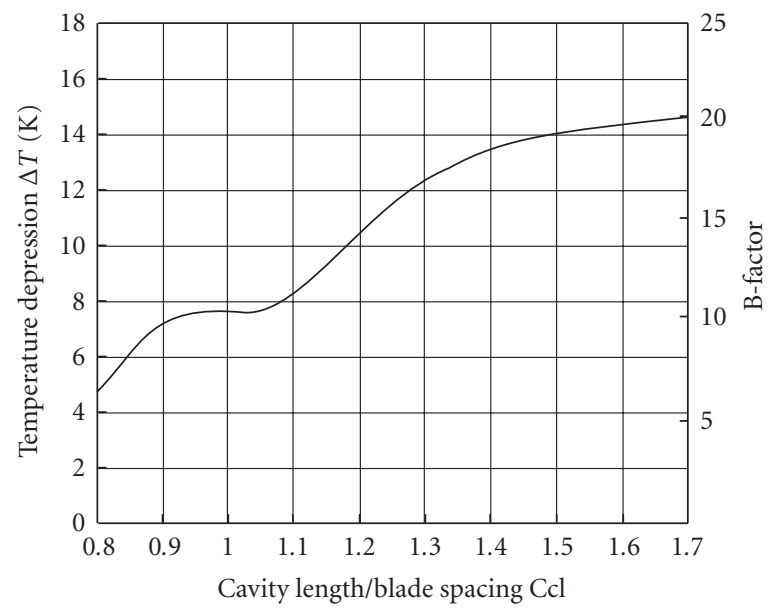

(b) Temperature depression

FIgURE 5: Experimental results in the case of LN2 and cold water (Yoshida et al. [1]).

estimated the effective temperature depression using Franc's method with (24), which is plotted in Figure 5(b). As we can see from Figure 5(a), the development of the cavity is suppressed in the LN2 cases due to the thermodynamic effect of cavitation, which is similar to the cases with R-114 (Figure 3). In Figure 5(b), the effective temperature depression is plotted for longer cavitation in Yoshida's LN2 experiment than that in Franc's R-114 experiment, and we can see from this figure that the larger the cavity length becomes, the more the temperature depression also increases, which is similar to the case with R-114. However, when the cavity trailing edge reaches the throat section of the inducer (the cavity length equals to the pitch $h$ ), the temperature depression decreases slightly, which is probably due to the interaction between the cavity trailing edge and the flow around the leading edge

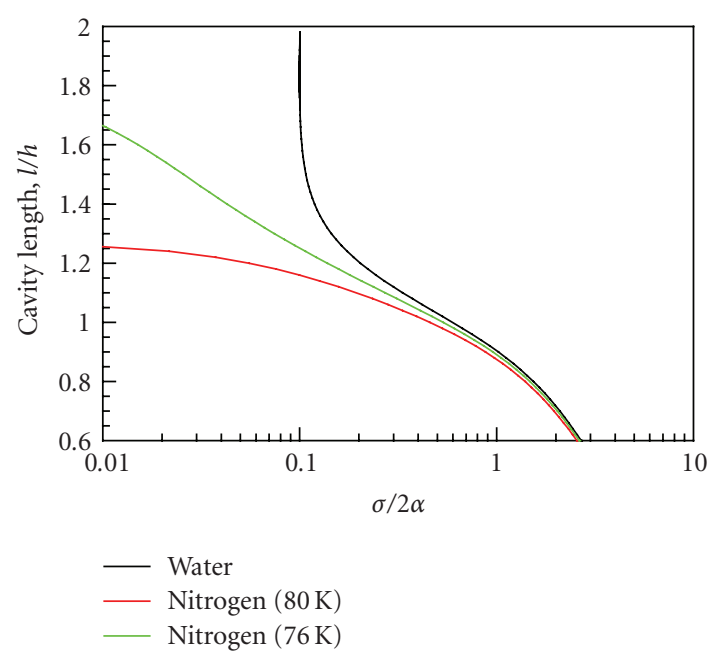

(a) Cavity length

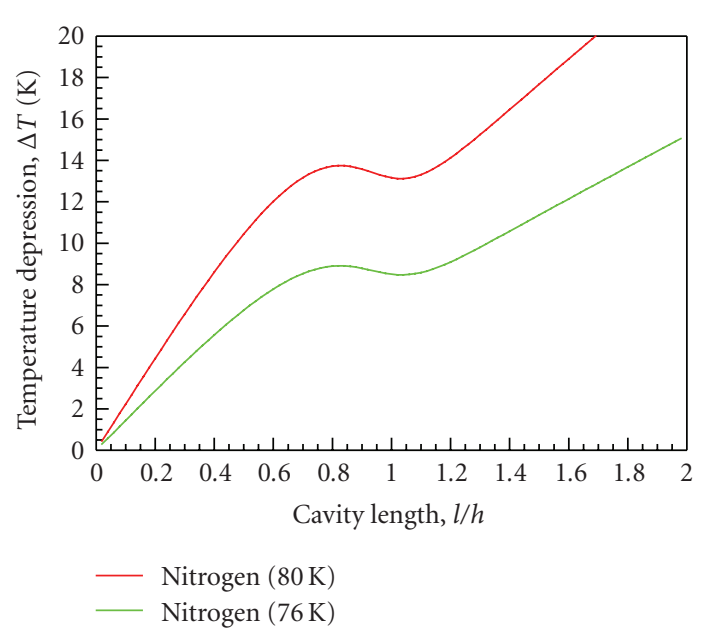

(b) Temperature depression

FIgURE 6: Numerical results in the case of LN2 and cold water.

of the adjacent blade. Further increase of the cavity length leads to the increase of the temperature depression again, whereas the temperature depression is being saturated when the cavity trailing edge approaches the trailing edge of the inducer blade. At that moment, the effective temperature depression is about $14 \mathrm{~K}$. Because the temperature range in the liquid nitrogen is between $63 \mathrm{~K}$ (triple point) and $126 \mathrm{~K}$ (critical point) and the test temperature is $80 \mathrm{~K}$, the value of $14 \mathrm{~K}$ is supposed to be almost the maximum possible temperature depression $(80-63=17 \mathrm{~K})$, which probably explains why the temperature depression seems to be limited in Figure 5(b).

Figure 6 shows the present analytical result for the LN2 with $76 \mathrm{~K}$ and $80 \mathrm{~K}$ and cold water cases. The analysis has been done for the cascade with the stagger angle of $75^{\circ}$, the solidity of 2.0. The incidence angle is set to be $5^{\circ}$ and the turbulence diffusion factor $\varepsilon$ is again set to be 1000 . We can see from the comparisons between Figures 5 and 6 that the present analysis can simulate the phenomenon 


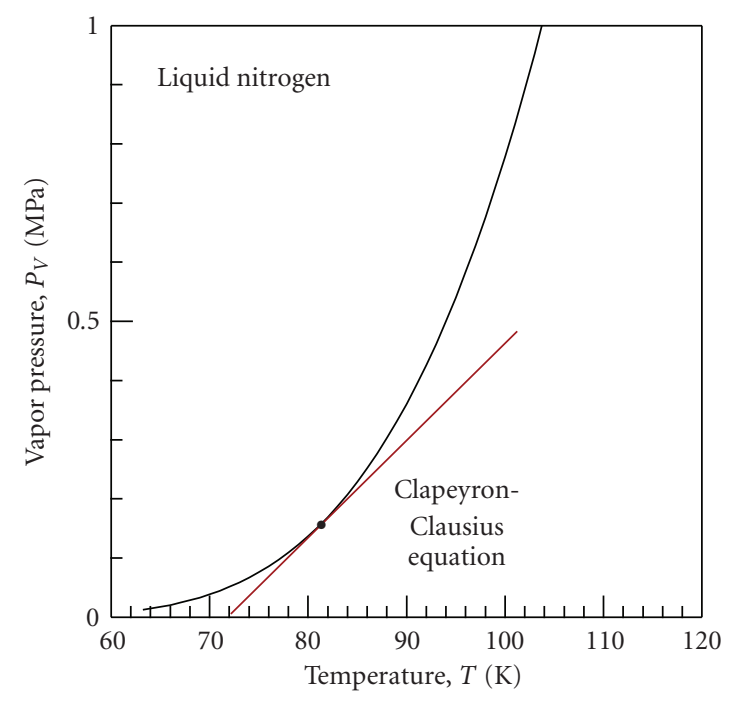

FIgURE 7: Saturation curve for nitrogen.

fairly well except that the effective temperature depression continues to increase even when the cavity trailing edge reaches the trailing edge of the blade in our analysis. This is probably because we have employed the ClapeyronClausius equation to estimate the vapor pressure for the local temperature, where the vapor pressure is assumed to change linearly with the change in the temperature; for the liquid nitrogen, the temperature range is narrow. The linear assumption is probably inappropriate in such cases.

Figure 7 shows the saturation curve in the nitrogen, from which we can find the large deviation of the ClapeyronClausius equation from the real saturation curve. Then, we made the analysis using the saturation curve in Figure 7 instead of (20). The results are shown in Figure 8. We can see the qualitatively good agreement between present analysis and the experiments shown in Figure 5. Then, in the cases with the fluids with narrower liquid temperature range and/or the cases where the large temperature depression is expected, the assumption of Clapeyron-Clausius equation as used in the estimate of B-factor seems to be insufficient to predict the thermodynamic effect of cavitation; at least, the real saturation curve should be taken into account for more qualitative/quantitative analysis of the thermodynamic effect of cavitation.

\section{CONCLUSIONS}

In the present study, we have carried out the singularity analysis considering the thermodynamic effect of cavitation for the cavitating cascade in Freon R-114 and liquid nitrogen. Through the detailed comparisons with the existing experiments done by Franc et al. [2] and Yoshida et al. [1], it is found that the present analysis can qualitatively well reproduce the thermodynamic effect of cavitation. The major conclusions obtained here are summarized as follows.

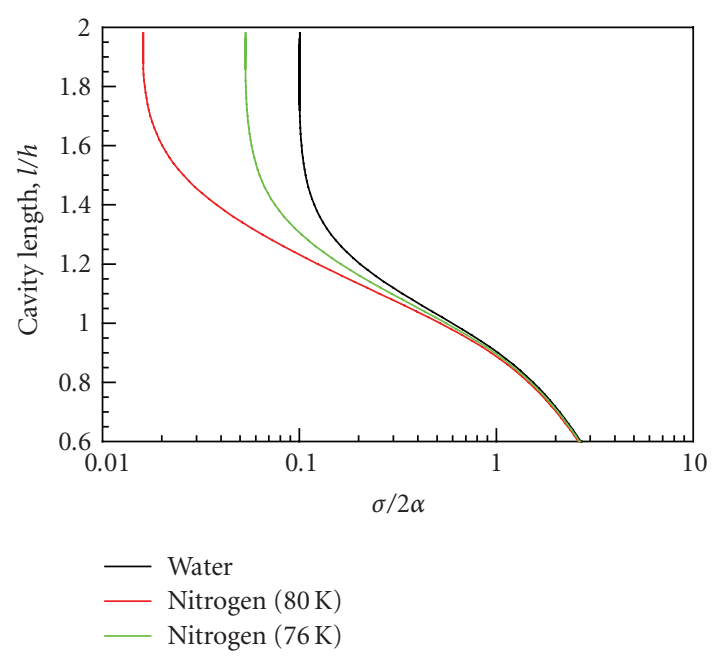

(a) Cavity length

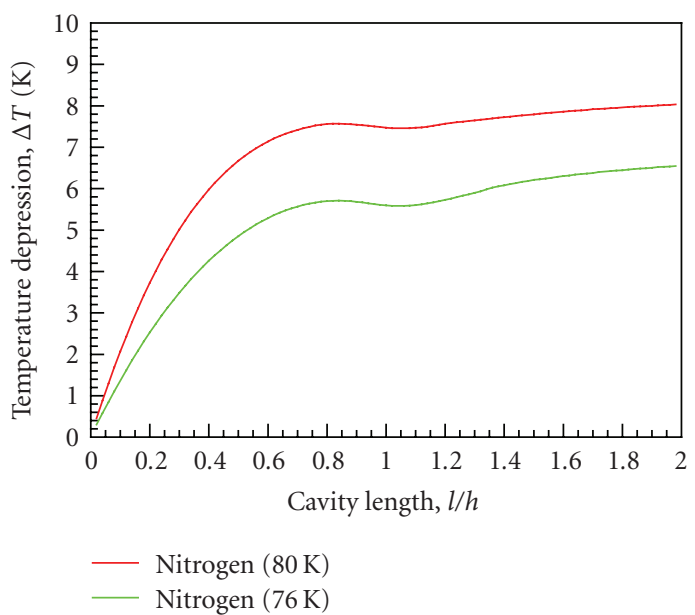

(b) Temperature depression

Figure 8: Modified results in the case of LN2 and cold water.

(1) The development of the cavity is suppressed due to the thermodynamic effect of cavitation. This effect is more significant for higher temperature.

(2) The thermodynamic effect becomes more apparent, and then the temperature depression becomes larger as the cavity becomes longer, because the larger amount of latent heat of evaporation is needed.

(3) When the cavity trailing edge reaches the throat section of the inducer, the temperature depression slightly decreases. This is probably due to the interaction between the cavity trailing edge and the flow around the leading edge of the adjacent blade.

(4) In the cases with the fluids with narrower liquid temperature range and/or the cases where the large temperature depression is expected, the temperature depression might be limited by the triple point. To simulate this effect, the real saturation curve should be at least taken into account. 
The present study analytically models the thermodynamic effect of cavitation, whereas some parts such as the determination of the turbulence diffusion factor $\varepsilon$ are still difficult. The quantitative estimates of them are crucial to develop this analysis for more practical applications. Further experimental data with cryogenic fluids and sophistication of the model are needed for this purpose. Moreover, the real flow in the cavitating inducer is so complicated with the tip leakage and the back flow cavitations in addition to the blade surface cavitation treated here. The analysis for the other forms of cavitation still remains for future study.

\section{NOMENCLATURE}

A: $\quad$ Coefficient matrix in (23)

a: $\quad$ Thermal diffusivity

B: $\quad$ Constant vector in (23)

C: Chordlength

$C_{q}, C_{\gamma 1}, C_{\gamma 2}$ : Normalized strength of singularities

$C_{P}$ : $\quad$ Specific heat

$C_{T}, C_{\theta}$ : Normalized temperature increase

$f: \quad \quad$ Kernel function, defined by (2)

$h$ : $\quad$ Blade spacing

$i$ : Imaginary unit

$K_{P}: \quad K_{P}=\varepsilon a / U$

L: $\quad$ Latent heat of evaporation

$l$ : Cavity length

$N$ : Number of blades

$n: \quad$ Blade index

$P_{V}: \quad$ Vapor pressure

Q: $\quad$ Unknown vector in (23)

q: $\quad$ Source representing cavity

$q_{T}: \quad$ Heat flux on cavity surface

T: $\quad$ Temperature

$U: \quad$ Main flow velocity

$V: \quad$ Local evaporation velocity

$u, v: \quad \quad \quad \quad$ low velocity components in $x$ and $y$ directions

$u_{c s}: \quad$ Velocity deviation on cavity surface

$u_{s}, v_{s}: \quad$ Velocity deviations in $x$ and $y$ directions

$w: \quad$ Complex conjugate velocity

$x, y: \quad$ Coordinates

$z: \quad$ Complex coordinate, $=x+i y$

$\alpha: \quad$ Angle of attack

$\beta$ : $\quad$ Stagger angle

$\gamma 1, \gamma 2$ : $\quad$ Bound vortices representing blade

$\Delta T: \quad$ Temperature depression

$\varepsilon: \quad$ Turbulent diffusion factor

$\eta: \quad$ Cavity thickness

$\lambda$ : $\quad$ Thermal conductivity

$\rho_{L}, \rho_{V}$ : Densities of liquid and vapor phases

$\sigma: \quad$ Cavitation number.

\section{ACKNOWLEDGMENT}

This study is partly supported by the Grant-in-Aid for Scientific Research for the Ministry of Education, Science, Sports and Culture (no. 19760119).

\section{REFERENCES}

[1] Y. Yoshida, K. Kikuta, S. Hasegawa, M. Shimagaki, and T. Tokumasu, "Thermodynamic effect on a cavitating inducer in liquid nitrogen," Journal of Fluids Engineering, vol. 129, no. 3, pp. 273-278, 2007.

[2] J.-P. Franc, C. Rebattet, and A. Coulon, "An experimental investigation of thermal effects in a cavitating inducer," Journal of Fluids Engineering, vol. 126, no. 5, pp. 716-723, 2004.

[3] A. J. Stepanoff, "Cavitation properties of liquids," Journal of Engineeing and Power, vol. 86, pp. 195-200, 1964.

[4] H. Kato, "Thermodynamic effect on incipient and developed sheet cavitation," in Proceedings of the International Symposium on Cavitation Inception, vol. 16, pp. 127-136, New Orleans, La, USA, December 1984.

[5] T. Tokumasu, Y. Sekino, and K. Kamijo, "A new modeling of sheet cavitation considering the thermodynamic effects," in Proceedings of 5th International Symposium on Cavitation (CAV'03), Osaka, Japan, November 2003, Cav03-GS-16-003.

[6] N. Tani and T. Nagashima, "Numerical analysis of cryogenic cavitating flow on hydrofoil-comparison between water and cryogenic fluids," in Proceedings of the 4th International Conference on Launcher Technology, Liege, Belgium, December 2002.

[7] A. Hosangadi, V. Ahuja, R. J. Ungewitter, and J. Busby, "Numerical study of a flat plate inducer: comparison of performance in liquid hydrogen and water," in Proceedings of the 42nd AIAA/ASME/SAE/ASEE Joint Propulsion Conference \& Exhibit, pp. 7210-7222, Sacramento, Calif, USA, July 2006, AIAA-2006-5070.

[8] J. Hord, "Cavitation in liquid cryogens: II-hydrofoil," NASA Contractor Report CR-2156, NASA, Washington, DC, USA, 1973.

[9] S. Watanabe, Y. Tsujimoto, and A. Furukawa, "Theoretical analysis of transitional and partial cavity instabilities," Journal of Fluids Engineering, vol. 123, no. 3, pp. 692-697, 2001.

[10] S. Watanabe, K. Sato, Y. Tsujimoto, and K. Kamijo, "Analysis of rotating cavitation in a finite pitch cascade using a closed cavity model and a singularity method," Journal of Fluids Engineering, vol. 121, no. 4, pp. 834-840, 1999.

[11] S. Watanabe, T. Hidaka, H. Horiguchi, A. Furukawa, and Y. Tsujimoto, "Steady analysis of the thermodynamic effect of partial cavitation using the singularity method," Journal of Fluids Engineering, vol. 129, no. 2, pp. 121-127, 2007.

[12] H. Horiguchi, S. Watanabe, Y. Tsujimoto, and M. Aoki, "A theoretical analysis of alternate blade cavitation in inducers," Journal of Fluids Engineering, vol. 122, no. 1, pp. 156-163, 2000.

\section{SUBSCRIPTS}

$n$ : Blade index

$\infty$ : Upstream infinity. 

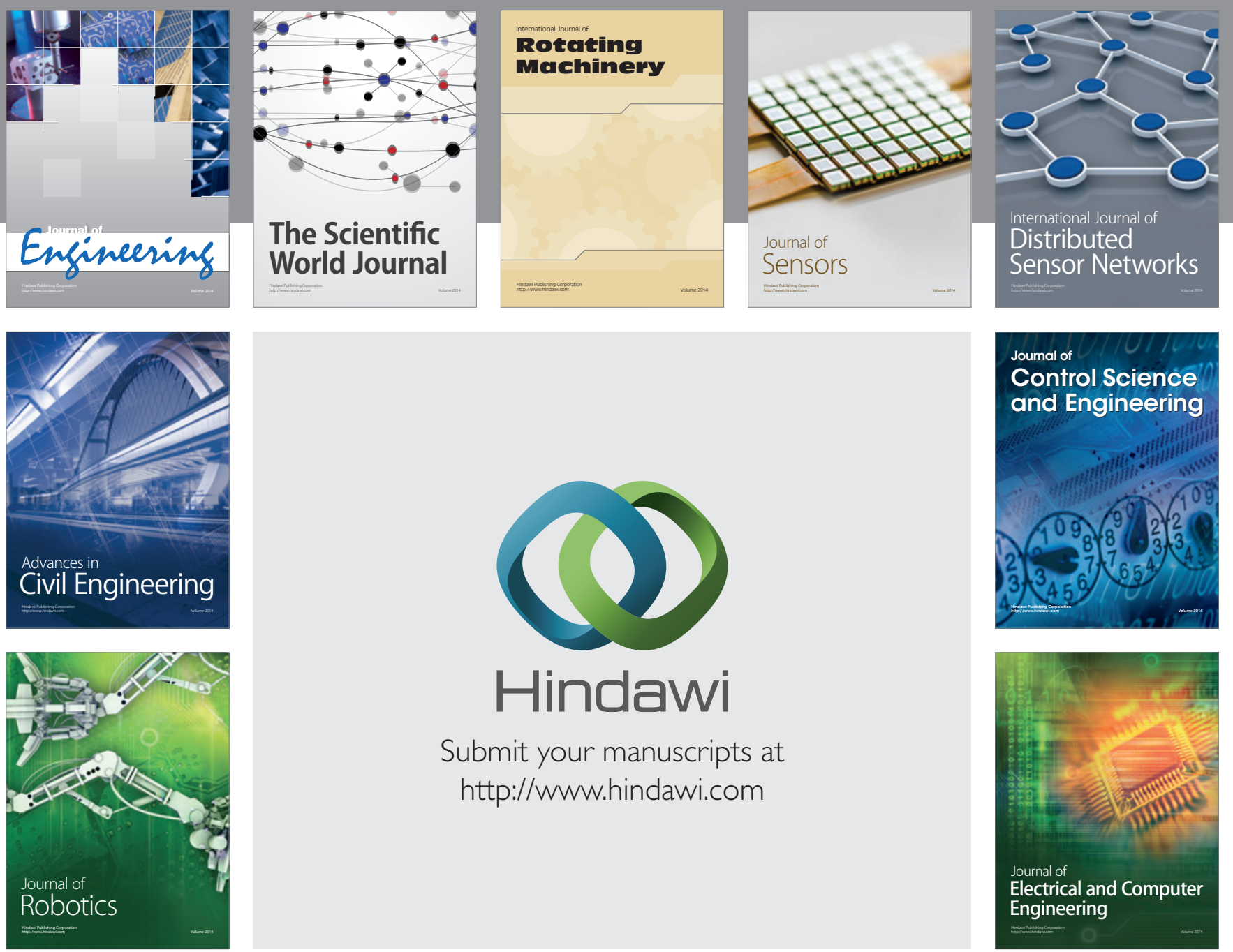

Submit your manuscripts at

http://www.hindawi.com
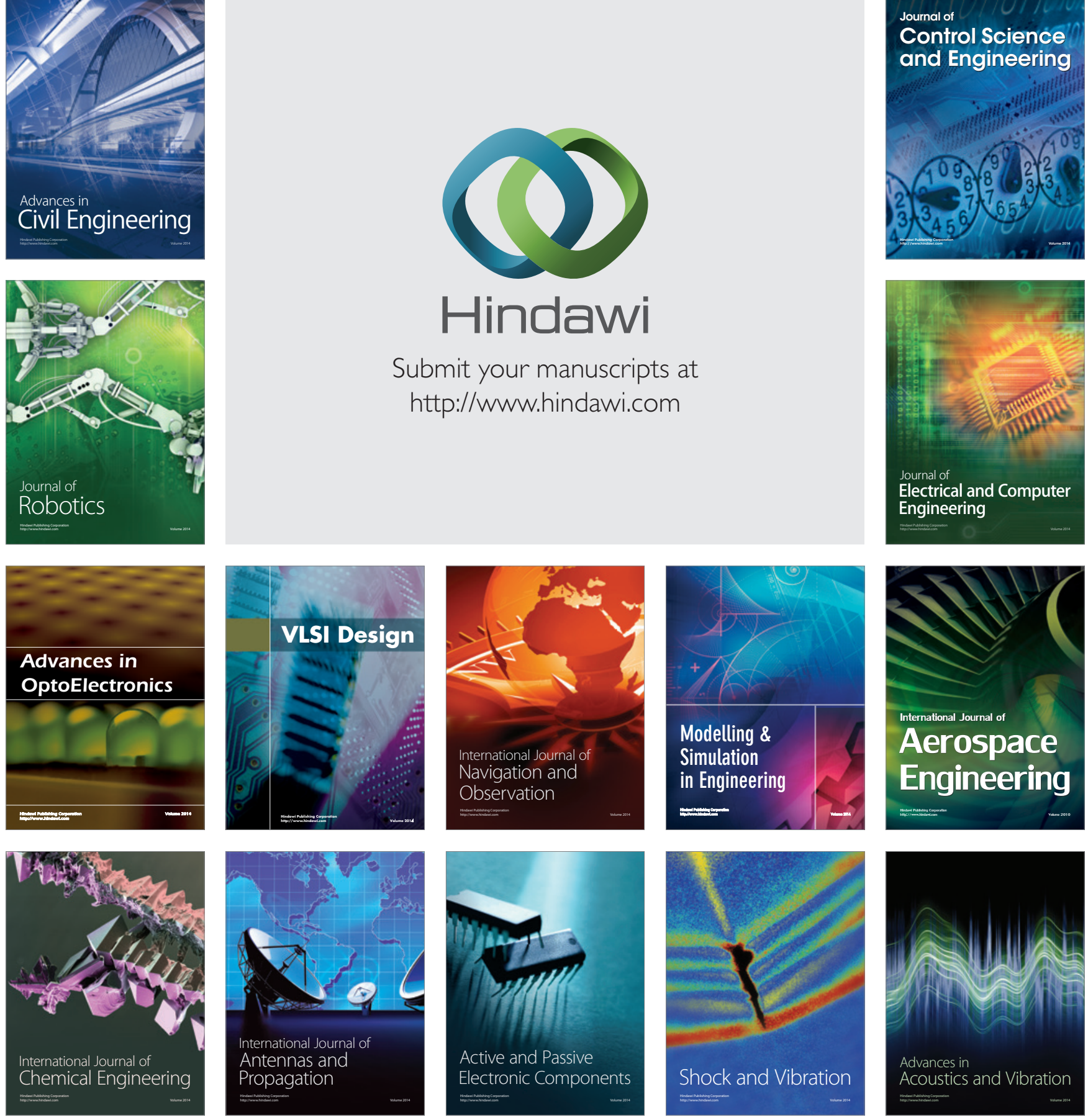